\title{
Tenth International Conference on Near-Field Optics, Nanophotonics, and Related Techniques (NFO 10)
}

\author{
Thomas Schmid
}

Published online: 22 November 2008

(C) Springer-Verlag 2008

The First International Conference on Near-Field Optics (NFO) took place in Arc-et-Senans (France) in 1992, eight years after the first scanning near-field optical microscopy (SNOM) experiments with visible light by Dieter Pohl et al. at IBM Zurich Research Laboratory in Rüschlikon (Switzerland). Since then this field has been growing and has extended into different new topics, for example apertureless probes, plasmonics, metamaterials, and nanoantennas. The conference is held every two years, at different locations worldwide. In 2006, near-field optics was "coming home" to Switzerland with NFO 9 held at EPFL in Lausanne. Two years later, the conference has moved to South America. NFO 10 was organized by Oscar E. Martínez and Andrea Bragas from the Universidad de Buenos Aires and took place at Salguero Plaza in Buenos Aires (Argentina) from September 1st-5th, 2008. During these five days, a school on near-field optics was held and results from this field of research were presented in ca. 100 oral and 240 poster presentations.

\begin{abstract}
Aims and topics The initial idea of SNOM was to confine light by guiding it through a sub-wavelength-sized aperture located within a sub-wavelength distance (within the optical near field) from a sample. When this nano light source is scanned over the sample surface, optical microscopy and spectroscopy can be performed with a spatial resolution below the optical diffraction limit. The drawback of the
\end{abstract}

T. Schmid $(\bowtie)$

Department of Chemistry and Applied Biosciences, ETH Zurich,

Wolfgang-Pauli-Str. 10,

8093 Zurich, Switzerland

e-mail:schmid@org.chem.ethz.ch limited light throughput of aperture glass fiber probes was overcome by introducing apertureless probes, metallic nanostructures in the laser focus, which can locally enhance the optical signal (e.g. Raman scattering or fluorescence). This effect can be obtained, for example, by exciting surface plasmon polaritons of the metal or by using enhancing nanoantennas. Nowadays, the NFO conference series covers a wide range of topics related to near-field optics from theory and fundamental principles to applications in physics, chemistry, biology, medicine, and materials science.

Scientific program For the first time, NFO opened with a one-day school on near-field optics. As Lukas Novotny said at the opening of the school, during the last sixteen years with nine NFO conferences, near-field optics has developed into a mature field of research, which gives us the opportunity to teach each other and learn from each other. The morning session held by Lukas Novotny was dedicated to the theory of light confinement by focusing optics and sub-wavelength apertures, and to the theory of light-matter interaction (e.g. Förster resonance energy transfer, FRET). In the afternoon session, Niek van Hulst gave a comprehensive overview on laboratory practice and applications of nanoscale optics and nanoantennas. The school closed with a talk by Peter Nordlander on plasmonic nanostructures.

The program of the conference itself consisted of three invited plenary lectures by Naomi J. Halas "Plasmonics: expanding the capabilities of near-field optics to molecular dimensions", Mario Marconi "Holographic nano-imaging and nanopatterning with table-top EUV lasers", and Xiang Zhang "Optical metamaterials, nano plasmonics, and superlens" and 22 sessions (all of two sessions held in parallel) with keynote lectures and contributed talks on the 
topics quantum systems, cavity nano optics (two sessions), plasmonics (three sessions), antennas (two sessions), IR and $\mathrm{THz}$ (two sessions), nano imaging (two sessions), biology and chemistry, photothermal, near-field enhancement (three sessions), instrumentation (two sessions), metamaterials (two sessions), and ultrafast phenomena. In addition, three poster sessions gave time for interesting discussions. In the poster hall there was also an exhibition where Attocube Systems, Coherent, Horiba Jobin Yvon, Nanonics Imaging, Newport, Olympus, RHK Technology, Spectra Physics, and Witec presented their latest products.

The program can be viewed at http://www.nfo10.org.

Conference highlights For this section, I have selected a few presentations and results with direct implications in analytical and bioanalytical chemistry. In her plenary lecture, Naomi J. Halas described nanoshells, which are able to locally enhance the electromagnetic field. Medical fluorescence imaging is often hampered by the low quantum yield of the contrast agents and their toxicity when applied at elevated concentrations. Naomi J. Halas described major improvement of image contrast by use of fluorescent dyes, which are chemically linked to enhancing nanoshells. Additionally, a strategy towards a new cancer therapy was presented, in which, after specific enrichment in tumor tissue, nanoshells are exposed to elevated laser powers in order to generate heat. A first clinical trial is already running.

Fritz Keilmann gave a talk and chaired a session on nearfield infrared and Terahertz imaging, which demonstrated the possibilities of material-specific contrast in highresolution images (down to $<10 \mathrm{~nm}$, irrespective of wavelength) with these techniques. New approaches overcome the limitations of single-wavelength measurements by using tunable radiation sources, for example free electron lasers (oral presentation by Susanne Kehr).

In her talk, Maria F. Garcia-Parajo reported on possibilities of developing aperture-type SNOM towards chemical imaging of biological cells in a liquid environment. She presented SNOM fluorescence imaging of a cell membrane with labeled lipid rafts and receptors. While the far-field images seemed to speak for a colocalization of receptors and lipid rafts, high-resolution SNOM images contradicted this postulate by revealing isolated receptors and lipid rafts. In a next step, the group will try to overcome the low light throughput of SNOM tips by growing an enhancing nanoantenna at the tip end. Such modified tips can optionally be functionalized with biomolecules in order to study non-covalent interactions.

Andreas Naber (oral and poster presentation) and Jörg Wissler (poster presentation) presented a combination of aperture-type SNOM and fluorescence correlation spectroscopy for study of the transport of molecules through nuclear pores. This method enables determination of the diffusion coefficient which describes the transport through a single membrane pore.

Apertureless tip-enhanced Raman spectroscopy (TERS) results were mainly presented (talks and posters) by representatives of the groups of Satoshi Kawata, Volker Deckert, and Renato Zenobi. The presentations ranged from new strategies to improve the spatial resolution to new applications in biology, for example nucleic acids, proteins, membranes, and biofilms.

The excursion on Wednesday, September 3rd led us to the Museo Nacional de Bellas Artes and gave a very interesting overview of Argentinean arts of the 19th and 20th century and insights into the history of Argentina. The day closed with the conference dinner at Salguero Plaza, where we came into contact with the - not only gastronomic - specialties of Buenos Aires and Argentina: fiery tango, delicious wines, and excellent beef.

Future and related conferences Traditionally, NFO is held every even year, followed by the International Conference on Surface Plasmon Photonics (SPP) in the odd years. SPP4 will be held in Amsterdam (The Netherlands) in 2009, and in 2010 we will say "Ni Hao" when we meet in Beijing (China) at NFO 11. 


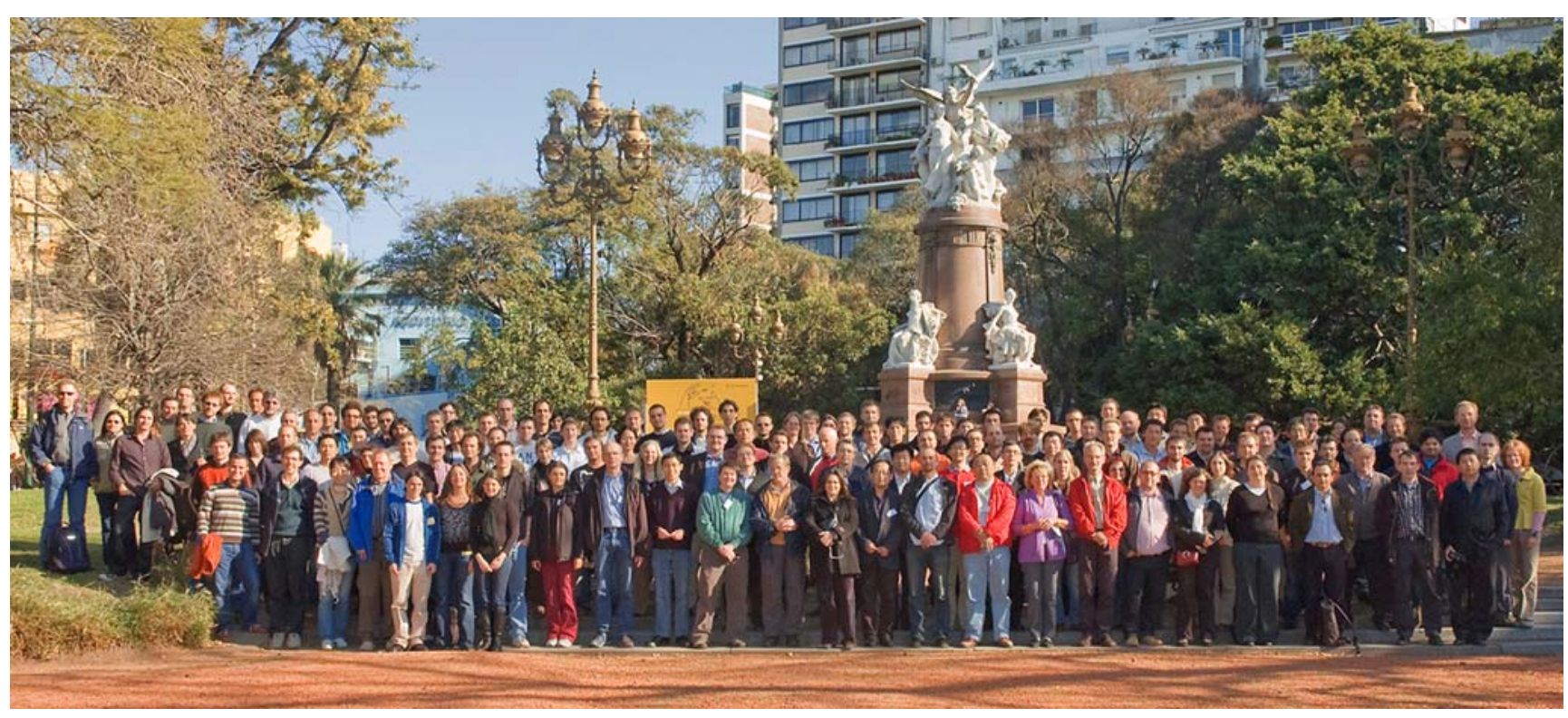

Official group photo taken next to the Museo Nacional de Bellas Artes in Buenos Aires (modified from http://www.nfo10.org)

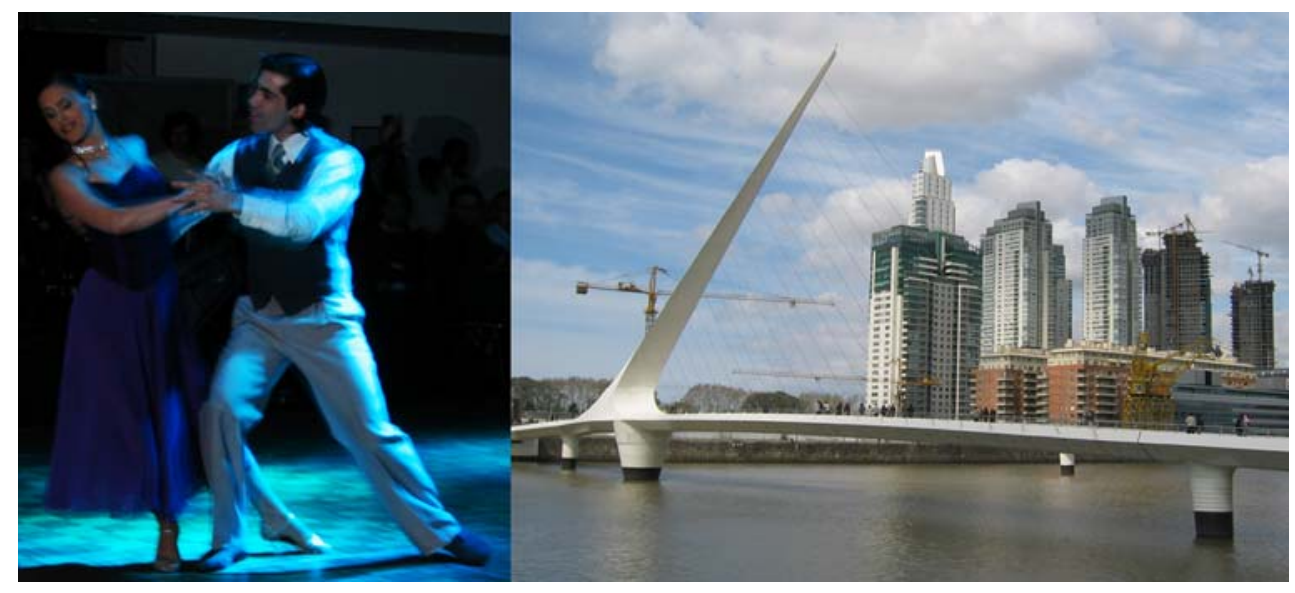

Impressions from the tango show at the conference dinner (left). This dance originated in Buenos Aires and inspired the Spanish architect Santiago Calatrava, when he designed the Puente de la Mujer (Woman's Bridge), located in the Puerto Madero district of Buenos Aires (right) 